\title{
Kapitalmarktreaktionen auf Rückschläge in der Produktentwicklung - Evidenz für pharmazeutische Unternehmen
}
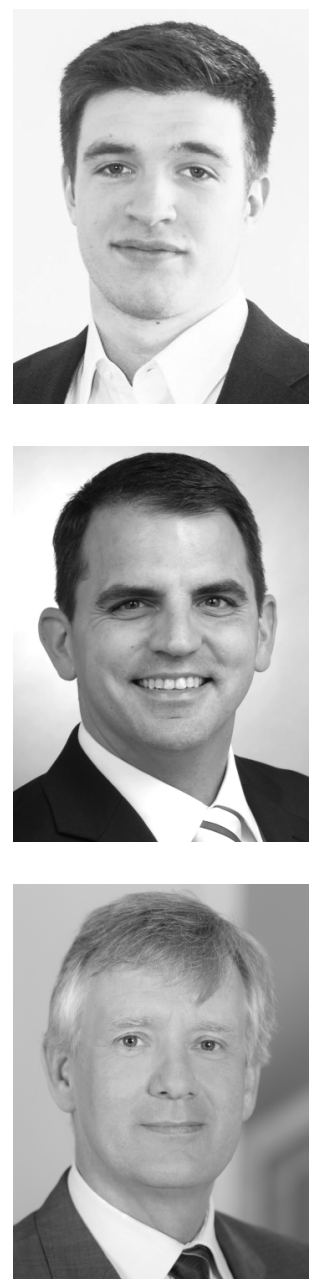

Niklas Kees, Jochen Panzer \& Dirk Schiereck

Neuproduktentwicklungen sind für ein forschendes Unternehmen regelmäßig mit erheblichen Risiken verbunden, die im Falle des Scheiterns auch Konsequenzen für die Kapitalmarktbewertung der Eigenkapitaltitel haben können. Im Vergleich zu anderen Branchen verläuft der Prozess der Neuproduktentwicklung in der pharmazeutischen Industrie sehr transparent, insbesondere auch mit Blick auf Rückschläge. Dadurch bietet sich hier die Gelegenheit, die Auswirkungen solcher Rückschläge, die in der Regel das (vorläufige) Ende eines kostspieligen Investitionsprozesses bilden, auf die Veränderung der Aktienkurswerte zu untersuchen. Je negativer eine Kapitalmarktreaktion ausfällt, umso stärkere Verluste erleiden die Eigenkapitalgeber. Ein enges Produktportfolio stellt hier ein besonderes Risiko dar, so dass sich solche Misserfolgsereignisse bei großen Pharmaunternehmen in der Kapitalmarktbewertung wesentlich schwächer auswirken als bei kleineren.

Zur Untersuchung der Kapitalmarktbewertung von veröffentlichten Rückschlägen wird eine Ereignisstudie und ein händisch zusammengestellter, neuer, sehr großer Datensatz mit 224 Rückschlagsankündigungen aus den Jahren 2010 bis 2017 genutzt. Im Ergebnis zeigen sich im Zeitraum der Ankündigungen extrem negative Aktienkursreaktionen von durchschnittlich $-23,7 \%$, wobei die Verluste sich nach Größe des Unternehmens unterscheiden. Zwar weisen sowohl kleine als auch große Unternehmen signifikant negative Werteffekte auf, aber der Effekt ist bei kleinen Unternehmen viel stärker. Potenzielle Erklärungsfaktoren für die Höhe und die Richtung des Werteffektes sind neben der Größe, gemessen als Marktkapitalisierung, u.a. der Return on Equity und die Frage der Entwicklung in Kooperation. Demnach lässt sich die Höhe des Werteffektes sowohl durch Unternehmenskennzahlen als auch durch den Inhalt der Veröffentlichung, bzw. den Status der Medikamentenentwicklung, erklären.

New product developments are regularly associated with considerable risks for a researchbased company, which, in the event of failure, can also have consequences for the capital market valuation of equity securities. Compared to other industries, the process of new product development in the pharmaceutical industry is very transparent, especially with regard to setbacks. This provides an opportunity to examine changes in share prices as an 
effect of such setbacks, which usually form the (temporary) end of a costly investment process. The more negative a capital market reaction is, the greater the losses suffered by equity investors. A narrow product portfolio represents a particular risk here, so that such failure events have a much weaker effect on the capital market valuation of large pharmaceutical companies than of smaller ones.

To analyze the capital market valuation of published setbacks, we use an event study and a hand-selected new, very large data set with 224 setback announcements from the years 2010 to 2017. As a result, we observe extremely negative share price reactions averaging $-23.7 \%$ in the period of the announcements, with losses varying according to the size of the company. Although both small and large companies show significant negative wealth effects, the effect is much stronger for small companies. In addition to size, measured as market capitalization, potential explanatory factors for the amount and direction of the value effect include return on equity and the development in cooperation. Accordingly, the amount of the wealth effect can be explained both by company key figures and by the content of the publication or the status of the drug development.

Neuproduktentwicklung, Pharmaforschung, Forschungsrückschlag, Ereignisstudie, Eigenkapitalkosten.

New product development, pharmaceutical R\&D, RひD setbacks, event study

\section{Motivation und Zielsetzung der Untersuchung}

Forschungs- und Entwicklungsausgaben bilden die Basis zum Aufbau immaterieller Vermögenswerte und neuer Produkte (Chauvin und Hirschey, 1993). Wenn als Ergebnis dieser Ausgaben Produktneueinführungen angekündigt werden, zeigen viele Studien, dass der Kapitalmarkt darauf insgesamt sehr positiv reagiert hat (bspw. Chaney et al., 1991; Lin und Chang, 20012). Neue Medikamente tragen in erheblichem Maße zu den Erlösen von Pharmaunternehmen bei (Grabowski und Kyle 2007; Grabowski und Vernon 2000). Allerdings fällt die Marktreaktion in forschungsintensiven Industrien, in denen regelmäßig neue Produkte erwartet werden, deutlich schwächer aus (Kelm et al., 1995). Zur Erklärung der positiven Kapitalmarktreaktionen verweisen $\mathrm{Hu}$ et al. (2013) auf strategische Flexibilitäten, die sich einem Management mit Produktneueinführungen bieten und die von den $\mathrm{Au}$ toren als Realoptionen modelliert werden. Je mehr und je werthaltigere Realoptionen ein Unternehmen besitzt, umso positiver fällt die Kapitalmarktreaktion auf eine Produktneueinführung und damit auf eine ausgeübte Realoption aus. Wenn langfristige Forschungsund Entwicklungsprozesse dagegen ohne erfolgreiche Produktneueinführung abgebrochen werden müssen und Realoptionen damit wertlos verfallen, ist dies spiegelbildlich zu den gelungenen Produktneueinführungen für die Eigentümer des betroffenen Unternehmens offensichtlich ein negatives Ereignis, das sich letztlich sogar auf die Zukunftsfähigkeit des Unternehmens und damit auf die Werthaltigkeit seiner Unternehmensanteile auswirken kann. Das relative Verlustpotential bei Fehlschlägen in der Produktentwicklung wird zudem mit der Unternehmensgröße und den damit verbundenen Diversifikationsmöglichkeiten einhergehen, wobei kleinere Unternehmen bei Fehlschlägen schneller in den Bereich existentieller Nöte geraten können, wie sie umgekehrt im Erfolgsfall stärker in ihrer Wertentwicklung profitieren (Chen und Ho 1997; Chen et al. 2002). 
Vor diesem Hintergrund erscheint die pharmazeutische Industrie besonders gut geeignet, den Zusammenhang zwischen Forschungsrückschlägen und Wertverlusten der Eigenkapitaltitel bei unterschiedlichen Unternehmensgrößen zu analysieren. Denn zum einen zeigt diese Industrie seit langem eine fortlaufende Tendenz zu steigenden Unternehmensgrößen durch Unternehmensübernahmen und Fusionen, was auch mit Risikotragfähigkeit und Vorteilen einer Diversifikation begründet wird (Schön, 2015). Zum anderen ist das Scheitern in der Produktentwicklung bei Pharmaunternehmen aufgrund des vergleichsweise sehr transparenten Forschungsprozesses mit drei Phasen klinischer Studien gut nachvollziehbar (Verband Forschender Arzneimittelhersteller e.V., 2015). Klinische Studien sind das Schlüsselelement in jedem Arzneimittelentwicklungsprozess. Nach erfolgter Freigabe durch die entsprechende Behörde wird in Phase I der neue Wirkstoff zunächst an gesunden Probanden getestet. Nach erfolgreichem Abschluss der Phase I folgt in Phase II ein Test mit erhöhter Anzahl an Probanden, wobei alle Teilnehmer von der Krankheit, gegen die der Wirkstoff entwickelt wurde, betroffen sind. In Phase III wird die Studie auf mehrere Tausend Probanden ausgeweitet, und es werden neben der Wirksamkeit auch Wechselwirkungen untersucht. Die Kosten der Durchführung steigen von Phase I zu Phase III stark an, so dass die Wahrscheinlichkeit der Marktzulassung mit jeder erfolgreich durchlaufenen Phase zwar steigt, aber auch der Verlust im Falle eines negativen Verlaufs exponentiell zunimmt.

Übertragen auf das Verständnis von strategischen Flexibilitäten und Produktneueinführungen als erfolgreich ausgeübte Realoptionen ist ein Scheitern im Arzneimittelentwicklungsprozess somit der wertlose Verfall einer Option, die bis zu ihrem Verfall umso höher bewertet wurde, je weiter der Entwicklungsprozess fortgeschritten und die Markteinführung wahrscheinlicher war. DiMasi et al. (2016) schätzen die kapitalisierten Durchschnittskosten in der Phase II auf 95 Mio. US-\$ und in der Phase III auf 314 Mio. US-\$. Konsequenterweise zeigt die bislang spärlich vorliegende empirische Evidenz zur Aktienbewertung von Pharmaunternehmen im Kontext gescheiterter klinischer Entwicklungsphasen, dass die Kapitalmarktreaktionen auf die Veröffentlichung von Fehlschlägen sehr negativ waren.

So untersuchen Deeds et al. (2003) die Auswirkung von 55 Entwicklungsabbrüchen im Stadium der Phasen I bis III und finden eine hoch signifikante Kapitalmarktreaktion von $-20,09 \%$, deren Variation im Datensatz u.a. durch die Unternehmensgröße erklärbar ist. Rothenstein et al. (2011) legen ein besonderes Augenmerk auf die Entwicklung der Aktienkurse vor dem Ereignis. Im Zeitraum von 2000 bis 2009 mit 23 positiven und 36 negativen Ereignissen halten sie als Ergebnis fest, dass vor der Bekanntgabe von positiven Studienergebnissen der Phase III der Kurs im Durchschnitt um 9,4\% steigt. Bei negativen Studienergebnissen fällt der Kurs dagegen um -4,6\%. Hwang (2013) nutzt einen stark beschränkten Stichprobenumfang von 16 positiven und 8 negativen Studienergebnissen der Phasen I bis III, die zwischen 2011 und 2013 veröffentlicht wurden. Dabei stammen die Ergebnisse ausschließlich von großen Unternehmen mit einem Mindestumsatz von $5 \mathrm{Mrd}$. US-Dollar pro Jahr aus Pharmazeutika. Der Medianwert der Kapitalmarktreaktionen für positive Studienergebnisse ist $0,8 \%$, für negative $-2,0 \%$. In beiden Fällen liegt statistische Signifikanz vor. Überprüfte Erklärungsfaktoren wie das Anwendungsgebiet, die Studienphase oder die Branche (Pharmazie oder Biotechnologie) zeigen keine statistische Signifikanz. 
Ähnliche Einsichten liefern Studien, die sich mit finalen Zulassungs- oder Ablehnungsentscheidungen der jeweiligen Aufsichtsbehörden befassen, insbesondere der FDA in den USA (Hamill et al. 2013). In einer der ersten Analysen in diesem Bereich identifizieren Bosch und Lee (1994) im Zeitraum 1962 bis 1989194 Genehmigungen und 18 Ablehnungen. Diese Stichprobe beschränkt sich jedoch nicht ausschließlich auf Medikamente, sondern umfasst auch andere medizinische Produkte, die der Prüfung der FDA bedürfen. Für die Unterstichprobe der Medikamentengenehmigungen wird eine hoch signifikant positive Aktienrendite von $1,75 \%$ berichtet, bei Ablehnungen ergeben sich negative -2,5\%. Sharma und Lacey (2004) berichten für 344 Genehmigungen und 41 Ablehnungen bei negativen Ereignissen eine hoch signifikant negative Rendite von -21,03\% und für positive Ereignisse ebenfalls hoch signifikante 1,56\%. Diese Ergebnisse werden insgesamt von Sarkar und De Jong (2006) bestätigt, wobei hier wie auch bei Rothenstein et al. (2011) die Kapitalmarktreaktionen eher noch heftiger ausfallen. Für die Zeit nach dem Markteintritt dokumentieren Ahmed et al. (2002) qualitativ ähnliche Werteffekte.

Der Überblick verdeutlicht zunächst die recht homogene Einsicht, dass Forschungsrückschläge signifikant negativ an den Kapitalmärkten aufgenommen worden sind, allerdings mit einer recht hohen Schwankungsbreite zwischen -2\% und -20\%. Dabei zeigt sich aber auch, auf welch dünner und schon recht alter Datenbasis der Erkenntnisstand zu den Kapitalmarktbewertungen von Fehlschlägen in der Produktentwicklung bei Pharmaunternehmen beruht, der zudem unter anderen Marktstrukturen und ungleich günstigeren Forschungs- und Entwicklungsaufwendungen pro Medikament ermittelt wurde. So schätzen DiMasi et al. (2003) für die frühen Studien die kapitalisierten Durchschnittskosten in der Phase II nur auf 38 Mio. US-\$ und in der Phase III auf gerade 45 Mio. US-\$. In der Phase III bedeutet dies eine nahezu Versiebenfachung des Aufwands zwischen 2003 und 2016. Diese dramatische Ausgabensteigerung lässt vermuten, dass die bereits dokumentierten negativen Werteffekte aus früheren Studien in den letzten Jahren ein noch weitaus größeres Ausmaß erreicht haben. Vor diesem Hintergrund wird nachfolgend auf der Basis eines aktuellen und im historischen Vergleich sehr großen Datensatzes zwei Hauptforschungsfragen nachgegangen:

1. Welchen Kursreaktionen waren die Aktionäre von Pharmaunternehmen in der jüngsten Vergangenheit im Schnitt bei Fehlschlägen in der Neuproduktentwicklung ausgesetzt?

2. Gibt es hierbei Größeneffekte mit heftigeren Kapitalmarktreaktionen für kleinere Unternehmen, wie sie umgekehrt bei erfolgreichen Produktneueinführungen beobachtet wurden (Chen und Ho 1997; Chen et al. 2002)?

Chen et al. (2002) beobachten, dass die Werteffekte bei erfolgreichen Produktneueinführungen auch von strategischen Interaktionen der ankündigenden Unternehmen innerhalb ihrer Industrie abhängen. Dementsprechend stellt sich im Falle eines tatsächlich zu beobachtenden Größeneffekts die bislang in diesem Kontext noch nicht adressierte Frage nach organisationalen Lösungen insbesondere für kleinere Unternehmen:

3. Kann die Verlustteilung durch strategische Interaktionen und die gemeinsame Forschung in einer Kooperation die negative Auswirkung eines Forschungsfehlschlags abmildern?

Zur Beantwortung dieser Fragen werden nachfolgend zunächst in Kapitel 2 und 3 Datensatz und Methodik erläutert. Daran schließen Kapitel 4, das der Vorstellung der Ergebnis- 
se der empirischen Analyse dient, und Kapitel 5 mit Erklärungsansätzen für die Ergebnisse an. Kapitel 6 schließt mit einer Diskussion und einem Ausblick.

\section{Zusammenstellung des Datensatzes}

Zur Identifizierung der Fehlschläge in der Produktentwicklung wird eine manuelle Internetrecherche durchgeführt. Dafür werden wie bei Rothenstein et al. (2011) auf Pharmaund Biotechnologie spezialisierte Internetseiten genutzt, namentlich BioSpace, BioCentury und Bionity. Als Suchworte dienen klinische Studie, Phase, Scheitern, Abbrechen und nicht erreicht. ${ }^{1}$ Der Zeitraum wird auf die Jahre 2010 bis 2017 begrenzt und schließt damit an den Zeitraum der vorherigen Studien an. Bei der Selektion findet keine Beschränkung der Nationalitäten statt. Die Generierung der Stichprobe ist in Abbildung 1 als Ablaufdiagramm dargestellt.

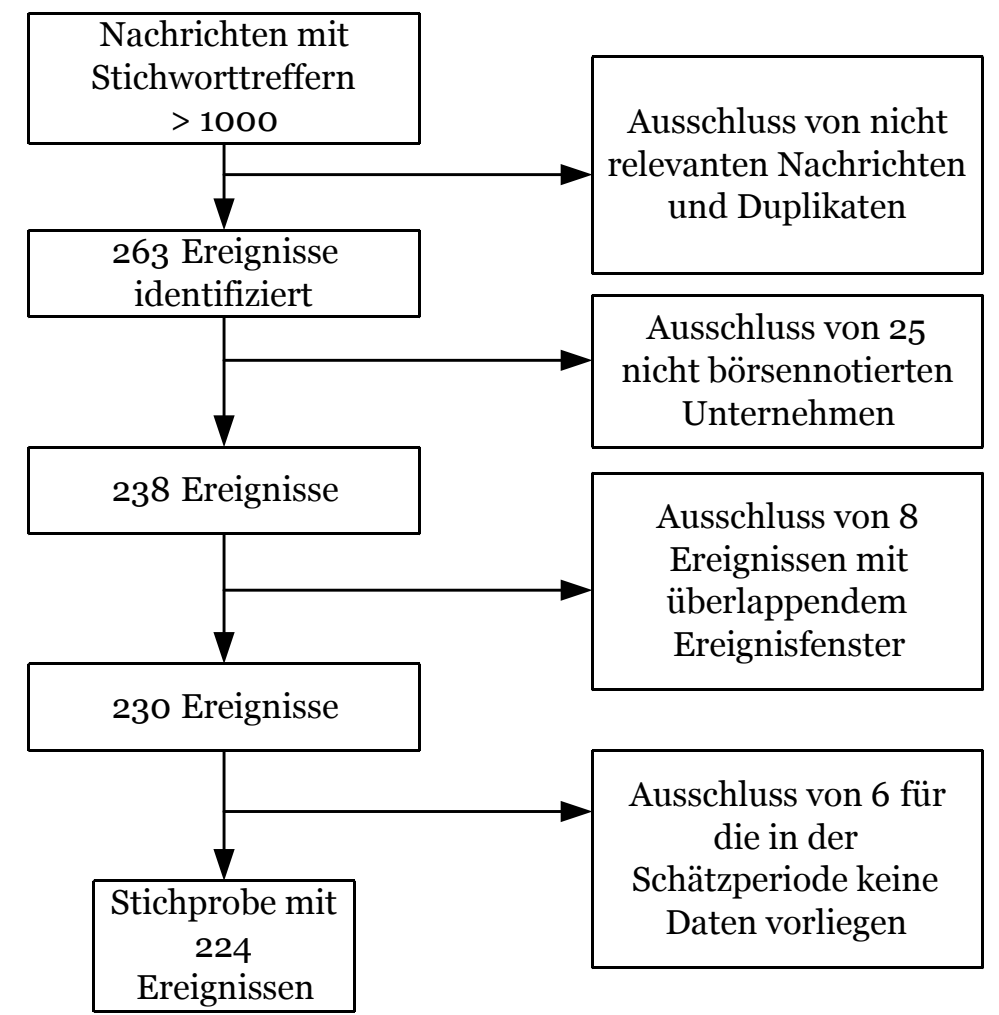

Abbildung 1: Ablaufdiagramm zur Generierung der Stichprobe.

Ein Datensatz von ursprünglich über 1.000 Einträgen wird manuell gesichtet und führt zu 263 Einträgen. Als Rückschlag werden dabei die folgenden Meldungen gewertet: Primärer Endpunkt nicht erreicht, Empfehlung des DMC zum vorzeitigen Abbruch, Meldungen zu Todesfällen mit Bezug zu der Studie, Beendigung der Entwicklung. Aufgrund von überlappenden Ereignisfenstern werden acht Ereignisse ausgeschlossen. 25 weitere Ereignisse müs-

1 Für die englischsprachigen Webseiten entsprechend die englischen Begriffe. 
sen ausgeschlossen werden, da die Unternehmen nicht börsennotiert sind. Für weitere sechs Ereignisse liegen für die benötigten Zeiträume keine Kursdaten vor. Dies führt zu einem finalen Datensatz von 224 Ereignissen, der aktueller und hinsichtlich des Stichprobenumfangs deutlich größer ist als die in bereits vorliegenden Studien.

Alle relevanten Kursdaten und Unternehmenskennzahlen werden den Datenbanken $D a$ tastream und Worldscope von Thomson Reuters entnommen. Zur Überprüfung des exakten Veröffentlichungsdatums werden sowohl die Unternehmenswebseiten als auch andere Nachrichtenquellen überprüft. Bei Veröffentlichungen, die nach Börsenschluss stattgefunden haben, wird der Folgetag als Ereignistag festgelegt.

Neben dem Ereignisdatum werden die Finanzgrößen Marktkapitalisierung, Umsatz, Cashflow, Return on Equity (RoE) und das Verhältnis aus Marktkapitalisierung und bilanziellem Eigenkapital (Market to Book Ratio - MtB) sowie folgende qualitative Informationen erhoben:

- Kooperation erfasst, ob das Medikament zusammen mit einem anderen Unternehmen entwickelt wurde. Die Form der Kooperation wird dabei nicht weiter spezifiziert. Inkludiert sind damit Forschungsallianzen, Lizensierungsprogramme und geteilte Vertriebsterritorien.

- Phase gibt an, in welcher Phase der klinischen Studien der Rückschlag des Medikaments zu verzeichnen ist.

- Blockbuster indiziert, ob ein Medikament Blockbuster-Potential hat. Dieses Kriterium wird als positiv deklariert, wenn die Umsatzprognosen über einer Milliarde US-Dollar liegen, es in den Nachrichten als Blockbuster-Kandidat bezeichnet wird oder entsprechende Äußerungen des Unternehmens vorliegen.

- Lead Drug bezeichnet die Bedeutung eines Medikaments für ein Unternehmen. Ein Medikament bekommt in der Datenerhebung den Status Lead Drug, wenn eine derartige Bezeichnung in den Nachrichten vorlag oder bei aktuellen Ereignissen dieser aus der Forschungspipeline geschlossen werden kann.

- Erstes Scheitern: Sind bis zum Zeitpunkt des Ereignisses keine negativen Studien zu dem Medikament veröffentlicht worden, so wird das Kriterium Erstes Scheitern als gegeben vermerkt.

- Zulassung gibt an, ob das Medikament bereits eine Marktzulassung (bspw. für andere Anwendung) erhalten hat.

- Interim wird als zutreffend vermerkt, wenn der Forschungsrückschlag aus einer Interimsanalyse hervorgeht.

- Beendigung: Wird am Ereignistag verkündet, dass die Entwicklung des Medikaments komplett eingestellt wird, dann wird Beendigung als zutreffend vermerkt. 
Tabelle 1: Deskriptive Statistik der Finanzkennzahlen im Datensatz

\begin{tabular}{|c|c|c|c|c|c|c|c|c|c|c|}
\hline & & 2010 & 2011 & 2012 & 2013 & 2014 & 2015 & 2016 & 2017 & Alle \\
\hline \multirow{6}{*}{ 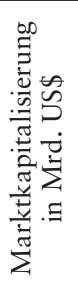 } & Max & 152,4 & 106,9 & 170,0 & 203,5 & 255,2 & 99,0 & 200,7 & 219,8 & 255,2 \\
\hline & $\varnothing$ & 63,8 & 49,8 & 37,9 & 60,9 & 56,1 & 23,0 & 31,5 & 39,4 & 45,9 \\
\hline & Median & 56,1 & 46,4 & 8,7 & 56,9 & 4,3 & 1,4 & 1,5 & 2,1 & 4,4 \\
\hline & Min & 0,0826 & 0,2108 & 0,0531 & 0,0651 & 0,0493 & 0,0005 & 0,0215 & 0,0214 & 0,0005 \\
\hline & Std. & 53,8 & 48,8 & 50,1 & 67,4 & 77,1 & 35,7 & 50,3 & 62,8 & 61,2 \\
\hline & Anzahl & 36 & 6 & 11 & 29 & 33 & 23 & 33 & 54 & 224 \\
\hline \multirow{6}{*}{ 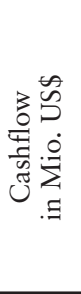 } & Max & 12855 & 6560 & 8705 & 9787 & 11649 & 4134 & 17146 & 9576 & 17146 \\
\hline & $\varnothing$ & 4525,8 & 1880,4 & 2133,1 & 3263,3 & 2760,3 & 686,7 & 1362,2 & 1540,6 & 2354,3 \\
\hline & Median & 4328,8 & 1213,5 & 680,0 & 2935,2 & $-6,7$ & $-6,6$ & $-19,0$ & $-17,3$ & 193,8 \\
\hline & Min & -162 & -104 & -22 & -181 & -245 & -1531 & -911 & -246 & -1531 \\
\hline & Std & 3875 & 2375 & 2679 & 3461 & 3985 & 1441 & 3616 & 2677 & 3487 \\
\hline & Anzahl & 35 & 6 & 11 & 29 & 32 & 21 & 33 & 53 & 220 \\
\hline \multirow{6}{*}{ 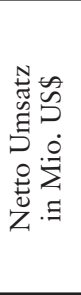 } & $\operatorname{Max}$ & 50009 & 44293 & 67425 & 58986 & 60869 & 35835 & 48458 & 49342 & 67425 \\
\hline & Mean & 25571 & 21638 & 13988 & 21522 & 17083 & 7971 & 7596 & 11499 & 15350 \\
\hline & Median & 27428 & 20567 & 11550 & 22603 & 1263 & 84 & 36 & 25 & 2190 \\
\hline & Min & 12,7 & 1,3 & 0,0 & 0,0 & 0,0 & 0,0 & 0,0 & 0,0 & 0,0 \\
\hline & Std. & 19935 & 21484 & 19206 & 21761 & 21550 & 11673 & 12160 & 17104 & 19259 \\
\hline & Anzahl & 35 & 6 & 11 & 29 & 32 & 21 & 33 & 53 & 220 \\
\hline \multirow{6}{*}{ 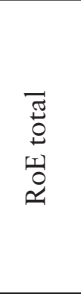 } & $\operatorname{Max}$ & 53,3 & 17,3 & 46,0 & 71,8 & 85,0 & 49,0 & 179,6 & 42,7 & 179,6 \\
\hline & Mean & 3,5 & $-8,9$ & $-9,6$ & $-9,6$ & $-106,2$ & $-18,7$ & $-42,6$ & $-58,9$ & $-37,6$ \\
\hline & Median & 16,2 & $-5,9$ & 5,5 & 10,1 & 7,1 & $-6,3$ & $-31,3$ & $-15,5$ & 6,5 \\
\hline & Min & $-381,8$ & $-48,9$ & $-73,6$ & $-160,2$ & $-1419,4$ & $-123,5$ & $-362,1$ & $-1959,0$ & $-1959,0$ \\
\hline & Std. & 72,5 & 25,4 & 47,4 & 60,7 & 354,3 & 48,3 & 91,1 & 277,3 & 197,2 \\
\hline & Anzahl & 34 & 6 & 11 & 28 & 29 & 17 & 33 & 48 & 206 \\
\hline \multirow{6}{*}{ 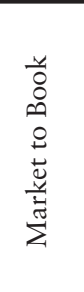 } & Max & 162,5 & 8,2 & 12,6 & 11,7 & 254,1 & 273,6 & 162,4 & 69,3 & 273,6 \\
\hline & Mean & 8,6 & 5,7 & 5,8 & 4,0 & 14,0 & 8,0 & $-2,3$ & 4,4 & 5,9 \\
\hline & Median & 2,7 & 6,6 & 4,1 & 3,1 & 4,0 & 3,8 & 3,5 & 3,4 & 3,4 \\
\hline & Min & 0,4 & 1,2 & 0,6 & $-25,0$ & $-39,3$ & $-189,8$ & $-331,0$ & $-58,7$ & $-331,0$ \\
\hline & Std. & 26,4 & 2,4 & 4,0 & 6,6 & 44,8 & 70,8 & 66,5 & 14,0 & 40,5 \\
\hline & Anzahl & 36 & 6 & 10 & 29 & 32 & 22 & 33 & 52 & 220 \\
\hline
\end{tabular}

Insgesamt sind 105 Unternehmen an den 224 Ereignissen beteiligt. Tabelle 1 bietet eine Übersicht über die erhobenen Finanzkennzahlen der Unternehmen, aufgegliedert nach Jahren. Dabei können Unternehmen mehrmals aufgeführt sein, da die Statistik sich an den Ereignissen orientiert, um möglichen Änderungen innerhalb des Jahres bezüglich der Marktkapitalisierung und der Market to Book Ratio gerecht zu werden. Anzahl gibt an, für wie viele Ereignisse sich die Daten in dem Jahr ermitteln lassen. Die Kennwerte zeigen eine breite Verteilung der Stichprobe. Die Marktkapitalisierung als wichtigster Größenindikator bei den einbezogenen Unternehmen liegt zwischen 490 Tsd. und 255,2 Mrd. US-Dol- 
lar. Die Umsätze variieren zwischen 0 und 67,43 Mrd. US-Dollar. Diese Umsatzlosigkeit ist nicht ungewöhnlich, da Biotechnologieunternehmen oft nur durch Verkauf, Meilensteinzahlungen, Umsatzbeteiligung oder Lizensierung von Medikamenten Umsätze erwirtschaften können. Im Durchschnitt haben die Unternehmen einen positiven Cashflow. Ein negativer Cashflow kommt vor allem bei den kleineren Unternehmen in der Stichprobe vor.

In Tabelle 2 werden die 224 Ereignisse hinsichtlich ihrer zeitlichen und geografischen Verteilung erläutert. Es sind 64 Rückschläge in Phase II und 148 Rückschläge in Phase III verkündet worden. Des Weiteren werden analog zu Hay et al. (2014) 5 Studien in der Phase I+II und 7 Studien in der Phase II+III jeweils zu den Phase II und Phase III Ereignissen gezählt. Insgesamt liegen damit 69 Ergebnisse aus Phase II und 155 Ergebnisse aus Phase III vor. Zu Phase I Studien gibt es nur zwei Publikationen, weshalb diese aus der Studie ausgeschlossen werden.

Tabelle 2: Deskriptive Statistik der Ereignisse unterteilt nach ausgewählten Kriterien

\begin{tabular}{|c|c|c|c|c|c|c|c|}
\hline & Spezifikation & $\begin{array}{l}\text { Anzahl der } \\
\text { Ereignisse }\end{array}$ & Anteil & & Spezifikation & $\begin{array}{l}\text { Anzahl der } \\
\text { Ereignisse }\end{array}$ & Anteil \\
\hline \multirow{4}{*}{ Phase } & II & 69 & $31 \%$ & \multirow{6}{*}{$\begin{array}{l}\text { Anwendungs- } \\
\text { gebiet }\end{array}$} & Onkologie & 83 & $37 \%$ \\
\hline & III & 156 & $69 \%$ & & Neurologie & 34 & $15 \%$ \\
\hline & & & & & Autoimmun & 32 & $14 \%$ \\
\hline & & & & & Kardiovaskulär & 17 & $8 \%$ \\
\hline \multirow{15}{*}{ Land } & USA & 111 & $49,30 \%$ & & Pulmologie & 9 & $4 \%$ \\
\hline & GB & 31 & $13,80 \%$ & & Sonstige & 50 & $22 \%$ \\
\hline & Schweiz & 21 & $9,30 \%$ & & & & \\
\hline & Deutschland & 14 & $6,20 \%$ & & & & \\
\hline & Frankreich & 9 & $4,00 \%$ & \multirow{2}{*}{ Kooperation } & $\mathrm{Ja}$ & 69 & $31 \%$ \\
\hline & Israel & 9 & $4,00 \%$ & & Nein & 156 & $69 \%$ \\
\hline & Japan & 9 & $4,00 \%$ & & & & \\
\hline & Dänemark & 8 & $3,60 \%$ & \multirow{8}{*}{ Jahre } & 2010 & 36 & $16 \%$ \\
\hline & Kanada & 4 & $1,80 \%$ & & 2011 & 6 & $3 \%$ \\
\hline & Schweden & 3 & $1,30 \%$ & & 2012 & 11 & $5 \%$ \\
\hline & Irland & 2 & $0,90 \%$ & & 2013 & 29 & $13 \%$ \\
\hline & Belgien & 1 & $0,40 \%$ & & 2014 & 33 & $15 \%$ \\
\hline & Niederlande & 1 & $0,40 \%$ & & 2015 & 23 & $10 \%$ \\
\hline & Bermuda & 1 & $0,40 \%$ & & 2016 & 33 & $15 \%$ \\
\hline & Australien & 1 & $0,40 \%$ & & 2017 & 54 & $24 \%$ \\
\hline
\end{tabular}

Mit 49,3\% stammen die meisten Verkündungen von US-amerikanischen Unternehmen. Danach folgen die europäischen Länder Großbritannien, Schweiz, Deutschland und 
Frankreich. Trotz der starken Gewichtung der USA ist eine große geografische Streuung im Datensatz vorhanden. Abgesehen von den Jahren 2011 und 2012 mit nur sehr wenigen Meldungen ist die zeitliche Verteilung über die Jahre recht homogen. 83 Ereignisse und damit $37 \%$ der Gesamtereignisse stammen aus dem Anwendungsgebiet der Onkologie.

\section{Methodik}

Die Messung der Konsequenzen aus den verkündeten Fehlschlägen auf die Finanzierungskosten erfolgt mittels einer Ereignisstudie. Dieses Instrument wurde auch in früheren ähnlichen Studien eingesetzt und gilt allgemein als etabliertes Werkzeug zur Quantifizierung der Auswirkungen von Ereignissen auf den Eigenkapitalwert betroffener Unternehmen. Der Tag der Ankündigung wird als Ereigniszeitpunkt mit $\mathrm{t}=0$ festgelegt, und es werden die Aktienkursbewegungen im Umfeld dieses Tages analysiert. Um zu ermitteln, welcher Teil der im Ereignisfenster beobachteten Kursbewegungen durch die Verkündung des Forschungsrückschlags begründet ist, wird eine hypothetische Kursentwicklung ohne Verkündung benötigt. Als Differenz der tatsächlich beobachteten Rendite und der ermittelten hypothetischen Rendite ergibt sich die abnormale Rendite (AR). Für die Ermittlung der hypothetischen Rendite ist das Marktmodell am verbreitetsten. Hier wird eine lineare Beziehung zwischen der Rendite einer einzelnen Aktie und einem Aktienmarktindex unterstellt, und über ein Schätzfenster vor dem Ankündigungstag wird der Zusammenhang berechnet; die Rendite $\mathrm{R}_{\mathrm{i}, \mathrm{t}}$ ergibt sich aus:

$R_{i, t}=\alpha_{i}+\beta_{i} R_{m, t}+\epsilon_{i, t}$

mit:

$\mathrm{R}_{\mathrm{i}, \mathrm{t}} \quad$ Rendite des Wertpapiers i zum Zeitpunkt t; $\alpha_{\mathrm{i}} \beta_{\mathrm{i}}$ Regressionsparameter für die Rendite des Wertpapiers i

$\mathrm{R}_{\mathrm{m}, \mathrm{t}} \quad$ Marktrendite des Aktiengesamtmarktes $\mathrm{m}$ zum Zeitpunkt $\mathrm{t} ; \epsilon_{\mathrm{i}, \mathrm{t}}$ Fehlerterm

Die abnormale Rendite wird sodann über verschiedene Zeiträume additiv zur kumulierten abnormalen Rendite (CAR) zusammengefasst (Fama et al.; 1969). Dazu werden die abnormalen Renditen für ein einzelnes Unternehmen i über einen bestimmten Zeitraum, das Ereignisfenster, kumuliert. $\tau_{1}$ ist der Start- und $\tau_{2}$ der Endzeitpunkt des Ereigniszeitfensters.

$\operatorname{CAR}_{i}\left(\tau_{1}, \tau_{2}\right)=\sum_{\tau=\tau_{1}}^{\tau_{2}} A R_{i, \tau}$

Die Aggregation der $\mathrm{CAR}_{\tau}$ über eine größere Gruppe von Beobachtungen ergibt die kumulierten durchschnittlichen abnormalen Renditen (CAAR):

$\operatorname{CAAR}\left(\tau_{1}, \tau_{2}\right)=\sum_{\tau=\tau_{1}}^{\tau_{2}} A A R_{\tau}$

Um die statistische Signifikanz der CAAR zu überprüfen, werden sowohl parametrische als auch nicht parametrische Tests genutzt. Als parametrischer Test wird hier der t-Test gezeigt. Der Corrado Rank Test und der Sign Test werden als nichtparametrische Tests durchgeführt. ${ }^{2}$ Als Ereignistag wird der Veröffentlichungstag der Bekanntgabe definiert.

2 Die Tests nach Patell (1976) sowie Boehmer et al. (1991) konnten keine weiteren Erkenntnisse bieten. Die entsprechenden Ergebnisse sind auf Anfrage erhältlich. 
Um diesen Ereignistag $(t=0)$ wird nachfolgend ein 41-tägiges Ereignisfenster von $t=-20$ bis $\mathrm{t}=20$ untersucht. Das Schätzfenster zur Ermittlung der hypothetischen Rendite wird wie in vergleichbaren Studien mit 120 Tagen bemessen, es beginnt bei $t=-141$ und endet bei $\mathrm{t}=-21$. Um der internationalen Zusammensetzung des Datensatzes angemessen Rechnung zu tragen, wird als Marktindex der MSCI World Index genutzt, der die Kursentwicklung von über 1.600 Unternehmen aus weltweit 23 Ländern abbildet. ${ }^{3}$ Die benötigten Zeitreihendaten von 2009 bis 2017 werden über Datastream bezogen.

\section{Ergebnisse der Ereignisstudie}

Im Folgenden werden die Ergebnisse der Ereignisstudie präsentiert. Wie der Verlauf der CAAR über das gesamte Ereignisfenster, der in Abbildung 2 dargestellt ist, zeigt, ist um den Ereignistag ein sehr negativer Werteffekt zu beobachten. Eine Antizipation ist nicht erkennbar. In den Folgetagen nach dem Ereignis hält die negative Entwicklung an und die Unternehmen verlieren nochmals ca. $5 \%$ an Wert.

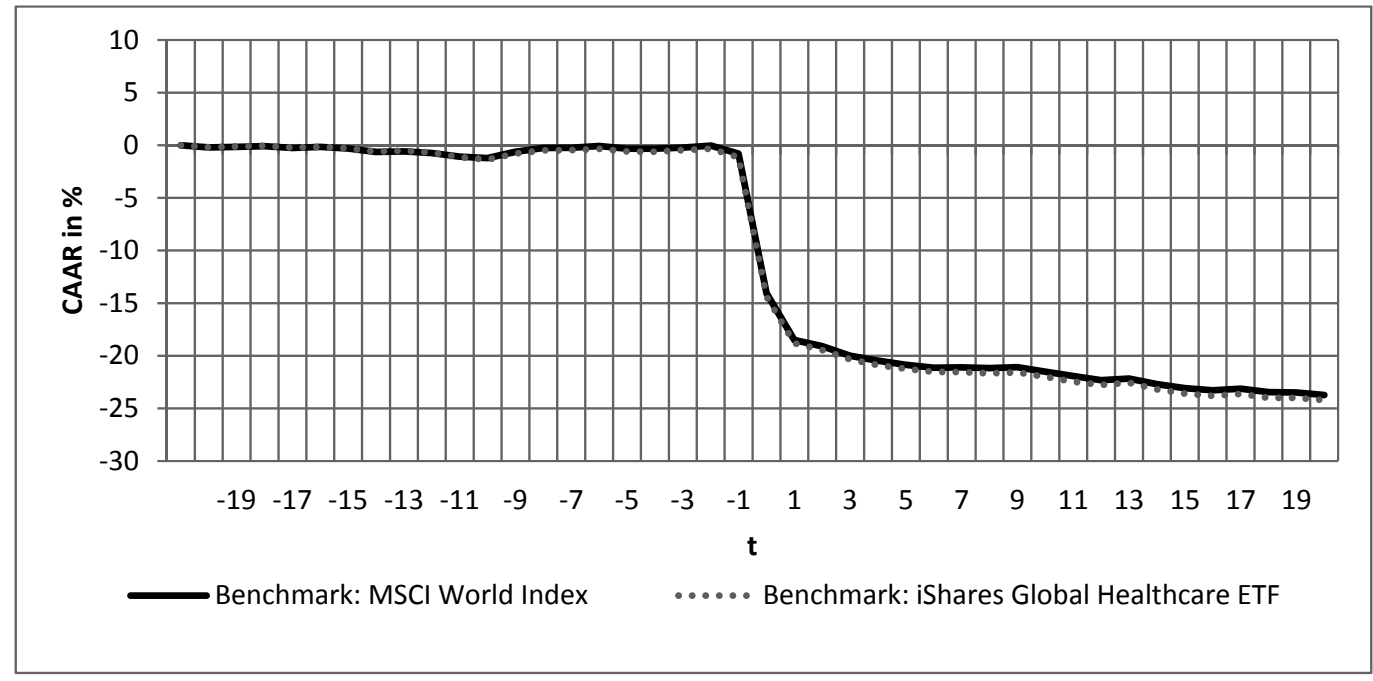

Abbildung 2: CAAR um den Ankündigungstag der Forschungsrückschläge für 224 Ereignisse.

Um die Robustheit der Ergebnisse zu testen, wird bei gleichen Parametern ein ETF ${ }^{4}$ als Benchmark genutzt. Der iShares Global Healthcare ETF umfasst eine große Anzahl der in dieser Arbeit vertretenen Unternehmen, wobei keines der Unternehmen einen Anteil von über $5 \%$ am ETF hat. ${ }^{5}$ Der Verlauf der CAAR ist ebenfalls in Abbildung 2 dargestellt und deckt sich mit dem Verlauf, der sich mit dem MSCI World Index als Benchmark ergibt. Die Ergebnisse werden also nicht durch die Benchmark getrieben. Für nachfolgende ARBerechnungen wird der MSCI World Index als Benchmark genutzt. Um die Wertentwick-

3 Vgl. MSCI (2017).

4 Exchange-traded fund/börsengehandelter Fond.

5 Vgl. Black Rock (2018). 
lung detaillierter zu illustrieren, werden in Tabelle 3 zunächst für den Gesamtdatensatz die CAAR nochmals über verschiedene Unterzeitfenster betrachtet.

Tabelle 3: CAAR um den Ankündigungstag inklusive statistischer Signifikanztests für 224 Ereignisse.

\begin{tabular}{ccccccccc}
\hline Tage & CAAR & Pos Neg & t-Test & p-Wert & $\begin{array}{c}\text { Corrado } \\
\text { Rank }\end{array}$ & p-Wert & Sign Test & p-Wert \\
\hline$(-20 \ldots 20)$ & $-0,2372$ & $62: 162$ & $-15,97$ & 0,000 & $-3,80$ & 0,000 & $-6,18$ & 0,000 \\
$(-10 \ldots 10)$ & $-0,2042$ & $68: 156$ & $-19,21$ & 0,000 & $-3,58$ & 0,000 & $-5,38$ & 0,000 \\
$(-5 \ldots 5)$ & $-0,2079$ & $50: 174$ & $-27,02$ & 0,000 & $-5,05$ & 0,000 & $-7,79$ & 0,000 \\
$(-1 \ldots 1)$ & $-0,1848$ & $56: 168$ & $-45,99$ & 0,000 & $-6,01$ & 0,000 & $-6,98$ & 0,000 \\
$(0 \ldots 0)$ & $-0,1324$ & $53: 171$ & $-57,09$ & 0,000 & $-7,57$ & 0,000 & $-7,39$ & 0,000 \\
$(-2 \ldots 0)$ & $-0,1384$ & $58: 166$ & $-34,44$ & 0,000 & $-4,96$ & 0,000 & $-6,72$ & 0,000 \\
$(-20 \ldots-2)$ & $-0,0003$ & $108: 116$ & $-0,03$ & 0,974 & $-0,07$ & 0,948 & $-0,03$ & 0,975 \\
$(3 \ldots 20)$ & $-0,0463$ & $89: 135$ & $-4,70$ & 0,000 & $-2,91$ & 0,004 & $-2,57$ & 0,010 \\
\hline
\end{tabular}

Im längsten Ereignisfenster [-20; 20] erreichen die CAAR -23,2 \% und sind statistisch hoch signifikant. Sowohl der parametrische t-Test als auch die nichtparametrischen Tests zeigen p-Werte, die mindestens kleiner als 0,0005 sind, und nur 62 Beobachtungen weisen positive CAR auf gegenüber 162 negativen (Pos Neg). Mit Blick auf die erste Forschungsfrage lässt sich somit feststellen, dass ein Scheitern bei der Neuproduktentwicklung in der Pharmaindustrie - mit aller Vorsicht bezüglich der Grenzen einer direkten Vergleichbarkeit - in den letzten Jahren deutlich kritischere Konsequenzen gezeigt hat, als sie in älteren Studien von Rothenstein et al. (2011) und Deeds et al. (2003) ausgewiesen wurden. Der zu beobachtende Marktwertverlust der betroffenen Unternehmen macht durchschnittlich fast ein Viertel des Wertes aus. Eine Korrekturbewegung zur Wertaufholung, die als Indikator für eine kurzfristige Überreaktion interpretiert werden könnte, ist nicht zu erkennen, so dass der Wertverzehr als nachhaltig einzustufen ist.

Die Ergebnisse von Hwang (2013) mit sehr viel kleineren negativen Kursausschlägen bei publizierten Forschungsrückschlägen basieren auf einer Stichprobe, die nur große Unternehmen enthält. Vor diesem Hintergrund und mit Blick auf die besonders positiven Kapitalmarktreaktionen bei erfolgreichen Produktneueinführungen (Chen und Ho 1997; Chen et al. 2002), wird im nächsten Schritt zur Adressierung der zweiten Forschungsfrage die Größenabhängigkeit der Kapitalmarktreaktionen überprüft, indem die Ereignisstudie mit zwei Unterstichproben durchgeführt wird. Die erste enthält große, die zweite die kleinen Unternehmen, wobei der Median der Marktkapitalisierung zur Unterteilung herangezogen wird. Die durchgezogene Linie in Abbildung 3 zeigt den Verlauf der CAAR für große Unternehmen (USP1). Der Verlauf der CAAR der kleinen Unternehmen (USP2) ist gepunktet dargestellt.

Der Größeneffekt ist frappierend. Während die Kapitalmarktreaktionen mit -3,8\% im Ereignisfenster [-20; 20] bei den Forschungsrückschlägen der großen Unternehmen hier nur wenig stärker als bei Hwang (2013) ausfallen, erleiden die kleineren Unternehmen der USP2 mit -43,7 \% sehr große Kursverluste, die als existentiell bezeichnet werden können. Wie die Detaildaten der Tabelle 4 a weiter verdeutlichen, gibt es bei den großen Unternehmen zumindest Anzeichen für Antizipationseffekte bzw. ein vorzeitiges Durchsickern der 
Informationen über die Fehlschläge, was bei den kleineren Unternehmen nicht zu beobachten ist.

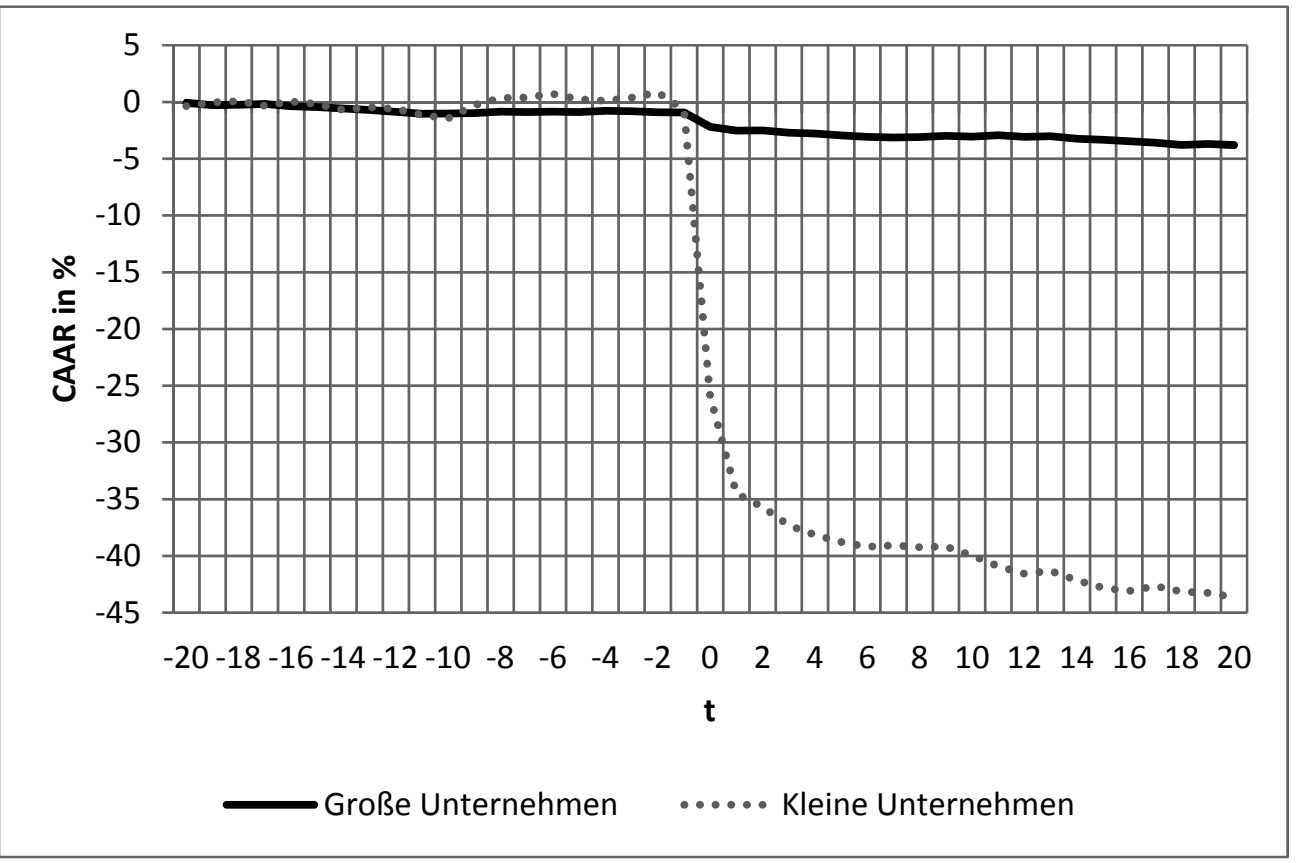

Abbildung 3: CAAR um den Ankündigungstag der Forschungsrückschläge für große und kleine Unternehmen.

Die Ergebnisse zeigen sich konsistent zu den positiven Größeneffekten bei erfolgreichen Produktneueinführungen und sind insgesamt als belastbarer Indikator eines Größeneffekts bei Forschungsfehlschlägen zu werten, der für kleinere Unternehmen so schwerwiegende Konsequenzen ausweist, dass nach Risikobegrenzungsmöglichkeiten gerade für diese kleineren Unternehmen zu suchen ist.

Tabelle 4a: CAAR um den Ankündigungstag inkl. statistischer Signifikanztests für große und kleine Unternehmen.

\begin{tabular}{|c|c|c|c|c|c|c|c|c|c|}
\hline & Tage & CAAR & Pos Neg & $\mathrm{t}$-Test & p-Wert & $\begin{array}{c}\text { Corrado } \\
\text { Rank }\end{array}$ & $\mathrm{p}$-Wert & Sign Test & p-Wert \\
\hline \multirow{8}{*}{ 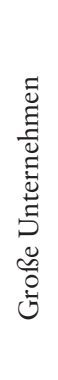 } & $(-20 \ldots 20)$ & $-0,0380$ & $43: 69$ & $-5,14$ & 0,000 & $-2,76$ & 0,006 & $-2,23$ & 0,026 \\
\hline & $(-10 \ldots 10)$ & $-0,0203$ & $56: 56$ & $-3,83$ & 0,000 & $-1,75$ & 0,080 & 0,23 & 0,819 \\
\hline & $(-5 \ldots 5)$ & $-0,0208$ & $44: 68$ & $-5,44$ & 0,000 & $-2,42$ & 0,015 & $-2,04$ & 0,041 \\
\hline & $(-1 \ldots 1)$ & $-0,0160$ & $45: 67$ & $-8,03$ & 0,000 & $-2,53$ & 0,012 & $-1,85$ & 0,064 \\
\hline & $(0 \ldots 0)$ & $-0,0127$ & $36: 76$ & $-10,96$ & 0,000 & $-4,13$ & 0,000 & $-3,55$ & 0,000 \\
\hline & $(-2 \ldots 0)$ & $-0,0137$ & $42: 70$ & $-6,86$ & 0,000 & $-3,19$ & 0,001 & $-2,42$ & 0,016 \\
\hline & $(-20 \ldots-2)$ & $-0,0091$ & $52: 60$ & $-1,81$ & 0,070 & $-0,86$ & 0,390 & $-0,53$ & 0,598 \\
\hline & $(3 \ldots 20)$ & $-0,0129$ & $46: 66$ & $-2,64$ & 0,008 & $-2,22$ & 0,027 & $-1,66$ & 0,097 \\
\hline
\end{tabular}




\begin{tabular}{|c|c|c|c|c|c|c|c|c|c|}
\hline & Tage & CAAR & Pos Neg & $\mathrm{t}$-Test & $\mathrm{p}$-Wert & $\begin{array}{c}\text { Corrado } \\
\text { Rank }\end{array}$ & p-Wert & Sign Test & $\mathrm{p}$-Wert \\
\hline \multirow{8}{*}{ 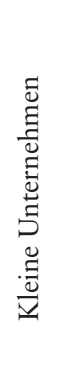 } & $(-20 \ldots .20)$ & $-0,4365$ & $19: 93$ & $-15,17$ & 0,000 & $-3,33$ & 0,001 & $-6,52$ & 0,000 \\
\hline & $(-10 \ldots 10)$ & $-0,3881$ & $12: 100$ & $-18,85$ & 0,000 & $-3,72$ & 0,000 & $-7,84$ & 0,000 \\
\hline & $(-5 \ldots 5)$ & $-0,3950$ & $6: 106$ & $-26,50$ & 0,000 & $-5,28$ & 0,000 & $-8,98$ & 0,000 \\
\hline & $(-1 \ldots 1)$ & $-0,3535$ & $11: 101$ & $-45,42$ & 0,000 & $-6,53$ & 0,000 & $-8,03$ & 0,000 \\
\hline & $(0 \ldots 0)$ & $-0,2522$ & $17: 95$ & $-56,13$ & 0,000 & $-7,58$ & 0,000 & $-6,89$ & 0,000 \\
\hline & $(-2 \ldots 0)$ & $-0,2630$ & $16: 96$ & $-33,79$ & 0,000 & $-4,64$ & 0,000 & $-7,08$ & 0,000 \\
\hline & $(-20 \ldots-2)$ & 0,0085 & $56: 56$ & 0,43 & 0,666 & 0,50 & 0,619 & 0,48 & 0,629 \\
\hline & $(3 \ldots 20)$ & $-0,0796$ & $43: 69$ & $-4,18$ & 0,000 & $-2,49$ & 0,013 & $-1,98$ & 0,048 \\
\hline
\end{tabular}

Auf eine umfassende Darstellung der Subsampleanalysen für Fehlschläge bei unterschiedlichen Anwendungsfeldern wird hier verzichtet. Die entsprechenden Ergebnisübersichten sind bei den Autoren erhältlich. Über alle Anwendungsklassen hinweg werden Fehlschläge am Kapitalmarkt mit hohen Wertkorrekturen eingepreist.

Tabelle 4b: CAAR um den Ankündigungstag inklusive statistischer Signifikanztests für Phase II und Phase III.

\begin{tabular}{|c|c|c|c|c|c|c|c|c|c|}
\hline & Tage & CAAR & Pos Neg & $\mathrm{t}$-Test & p-Wert & $\begin{array}{c}\text { Corrado } \\
\text { Rank }\end{array}$ & $\mathrm{p}$-Wert & Sign Test & p-Wert \\
\hline \multirow{8}{*}{ 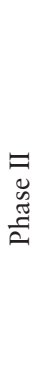 } & $(-20 \ldots 20)$ & $-0,3228$ & $17: 47$ & $-58,07$ & 0,000 & $-26,70$ & 0,008 & $-34,20$ & 0,001 \\
\hline & $(-10 \ldots 10)$ & $-0,2715$ & $16: 48$ & $-57,16$ & 0,000 & $-31,65$ & 0,002 & $-36,71$ & 0,000 \\
\hline & $(-5 \ldots 5)$ & $-0,2782$ & $11: 53$ & $-71,93$ & 0,000 & $-42,81$ & 0,000 & $-49,22$ & 0,000 \\
\hline & $(-1 \ldots 1)$ & $-0,2433$ & $16: 48$ & $-70,70$ & 0,000 & $-54,52$ & 0,000 & $-36,71$ & 0,000 \\
\hline & $(0 \ldots 0)$ & $-0,1640$ & $11: 53$ & $-59,40$ & 0,000 & $-64,77$ & 0,000 & $-49,22$ & 0,000 \\
\hline & $(-2 \ldots 0)$ & 0,0067 & $32: 32$ & 11,46 & 0,252 & 0,779 & 0,436 & 0,33 & 0,739 \\
\hline & $(-20 \ldots-2)$ & $-0,2392$ & $14: 50$ & $-53,88$ & 0,000 & $-16,79$ & 0,093 & $-41,71$ & 0,000 \\
\hline & $(3 \ldots 20)$ & $-0,0836$ & $26: 28$ & $-29,77$ & 0,003 & $-21,32$ & 0,033 & $-11,68$ & 0,243 \\
\hline \multirow{8}{*}{ 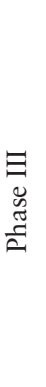 } & $(-20 \ldots 20)$ & $-0,1967$ & $39: 108$ & $-70,08$ & 0,000 & $-37,68$ & 0,000 & $-55,48$ & 0,000 \\
\hline & $(-10 \ldots 10)$ & $-0,1669$ & $47: 100$ & $-67,68$ & 0,000 & $-27,71$ & 0,006 & $-42,29$ & 0,000 \\
\hline & $(-5 \ldots 5)$ & $-0,1743$ & $42: 105$ & $-73,56$ & 0,000 & $-41,15$ & 0,000 & $-50,53$ & 0,000 \\
\hline & $(-1 \ldots 1)$ & $-0,1570$ & $39: 108$ & $-75,19$ & 0,000 & $-45,26$ & 0,000 & $-55,48$ & 0,000 \\
\hline & $(0 \ldots 0)$ & $-0,1227$ & $44: 103$ & $-64,84$ & 0,000 & $-60,93$ & 0,000 & $-47,23$ & 0,000 \\
\hline & $(-2 \ldots 0)$ & 0,0002 & $67: 80$ & 0,086 & 0,932 & $-0,571$ & 0,568 & $-0,929$ & 0,353 \\
\hline & $(-20 \ldots-2)$ & $-0,1659$ & $38: 109$ & $-74,36$ & 0,000 & $-24,20$ & 0,016 & $-57,13$ & 0,000 \\
\hline & $(3 \ldots 20)$ & $-0,0308$ & $59: 88$ & $-29,07$ & 0,004 & $-29,51$ & 0,003 & $-22,48$ & 0,025 \\
\hline
\end{tabular}

Bemerkenswerter fallen hingegen die Ergebnisse bei einer Unterteilung des Datensatzes in zwei Untergruppen nach der Phase der klinischen Studien aus, die Tabelle 4b zusammenfasst. Zum einen zeigt sich hier, dass in beiden Subsamples ein erheblicher Teil der Kursre- 
aktion bereits vor der offiziellen Verkündung der Forschungsrückschläge erfolgt, was insbesondere aus Sicht der Regulierung und Börsenaufsicht Anlass zu weiterführenden Untersuchungen bietet. Zum anderen fällt auf, dass die negativen Werteffekte bei Forschungsfehlschlägen in der Phase II insgesamt heftiger ausfallen als in der Phase III, was angesichts der Unterschiede in den Investitionssummen zwischen beiden Phasen zunächst verwundert. Eine mögliche Erklärung liegt wohl in der größeren Sichtbarkeit der Forschungsfortschritte für Außenstehende, wenn erst einmal die Phase III erreicht ist. Die Überraschung eines Scheiterns in Phase II wird dann gegenüber Rückschlägen in Phase III noch mehr ohne jede Vorwarnung und Antizipation eintreten. Es drängt sich aber für die zukünftige Forschung auf, diesen Befund noch tiefergehender zu analysieren. Der Einsatz von Fallstudien könnte dabei spannende Aufschlüsse bieten.

Tabelle 4c: CAAR um den Ankündigungstag inklusive statistischer Signifikanztests für USA und Rest der Welt.

\begin{tabular}{|c|c|c|c|c|c|c|c|c|c|}
\hline & Tage & CAAR & Pos Neg & $\mathrm{t}$-Test & $\mathrm{p}$-Wert & $\begin{array}{l}\text { Corrado } \\
\text { Rank }\end{array}$ & $\mathrm{p}$-Wert & Sign Test & $\mathrm{p}$-Wert \\
\hline \multirow{8}{*}{$\underset{\sim}{\mathscr{D}}$} & $(-20 \ldots 20)$ & $-0,3201$ & $56: 54$ & $-85,98$ & 0,000 & $-32,66$ & 0,001 & $-57,18$ & 0,000 \\
\hline & $(-10 \ldots 10)$ & $-0,2589$ & $26: 84$ & $-85,26$ & 0,000 & $-27,50$ & 0,001 & $-51,46$ & 0,000 \\
\hline & $(-5 \ldots 5)$ & $-0,2688$ & $44: 86$ & $-89,89$ & 0,000 & $-41,88$ & 0,000 & $-55,28$ & 0,000 \\
\hline & $(-1 \ldots 1)$ & $-0,2464$ & $25: 85$ & $-93,01$ & 0,000 & $-57,74$ & 0,000 & $-53,37$ & 0,000 \\
\hline & $(0 \ldots 0)$ & $-0,1767$ & $25: 85$ & $-76,48$ & 0,000 & $-72,83$ & 0,000 & $-53,37$ & 0,000 \\
\hline & $(-2 \ldots 0)$ & 0,0058 & $57: 53$ & 15,71 & 0,116 & 12,61 & 0,207 & 0,77 & 0,442 \\
\hline & $(-20 \ldots-2)$ & $-0,2594$ & $54: 56$ & $-86,03$ & 0,000 & $-24,29$ & 0,015 & $-59,09$ & 0,000 \\
\hline & $(3 \ldots 20)$ & $-0,0607$ & $46: 64$ & $-34,39$ & 0,001 & $-21,84$ & 0,029 & $-13,30$ & 0,184 \\
\hline \multirow{8}{*}{ 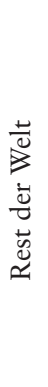 } & $(-20 \ldots .20)$ & $-0,1450$ & $38: 76$ & $-45,77$ & 0,000 & $-30,58$ & 0,001 & $-34,54$ & 0,000 \\
\hline & $(-10 \ldots 10)$ & $-0,1424$ & $40: 74$ & $-46,73$ & 0,000 & $-31,23$ & 0,001 & $-30,79$ & 0,000 \\
\hline & $(-5 \ldots 5)$ & $-0,1456$ & $33: 81$ & $-58,46$ & 0,000 & $-41,32$ & 0,000 & $-43,91$ & 0,000 \\
\hline & $(-1 \ldots 1)$ & $-0,1245$ & $31: 83$ & $-57,51$ & 0,000 & $-43,70$ & 0,000 & $-47,65$ & 0,000 \\
\hline & $(0 \ldots 0)$ & $-0,1640$ & $35: 79$ & $-59,40$ & 0,000 & $-64,77$ & 0,000 & $-49,22$ & 0,000 \\
\hline & $(-2 \ldots 0)$ & $-0,0018$ & $50: 64$ & $-0,839$ & 0,401 & $-15,33$ & 0,207 & $-12,06$ & 0,223 \\
\hline & $(-20 \ldots-2)$ & $-0,1162$ & $35: 79$ & $-47,23$ & 0,000 & $-14,17$ & 0,015 & $-40,16$ & 0,000 \\
\hline & $(3 \ldots 20)$ & $-0,0289$ & $47: 67$ & $-22,78$ & 0,023 & $-30,14$ & 0,029 & $-17,68$ & 0,077 \\
\hline
\end{tabular}

Eine Unterteilung nach der Herkunft der betroffenen Unternehmen in US-amerikanische Firmen, die knapp die Hälfte der Beobachtungen stellen, und Pharmaproduzenten aus dem Rest der Welt bietet hingegen, wie Tabelle $4 \mathrm{c}$ belegt, keine wesentlichen neuen Einsichten. Die Ergebnisse fallen in beiden Untergruppen sehr ähnlich aus.

\section{Erklärungsansätze für die Ereignisstudienergebnisse}

Die Ereignisstudien haben insgesamt sehr überzeugende Evidenz erbracht, dass Rückschläge in der Produktentwicklung bei Pharmaunternehmen mit sehr hohen negativen Werteffekten einhergehen, und damit unsere erste Forschungsfrage beantwortet. Die Subsample- 
betrachtungen haben zudem erste Anhaltspunkte zur zweiten Forschungsfrage ergeben und Hinweise auf einen Größeneffekt geliefert. Um diese indikative Beobachtung weiter zu unterlegen und zudem auch Forschungsfrage 3 zu adressieren, werden nachfolgend die kumulierten abnormalen Renditen (CAR $(-1,1)$ ) im Rahmen von multivariaten Regressionsanalysen mittels Kleinst-Quadrate-Schätzung auf mögliche Erklärungsfaktoren untersucht. Zunächst wird ein Basismodell, das nur die Kontrollvariablen enthält, getestet. Anschließend werden die unabhängigen Variablen jeweils einzeln in separaten Modellen mit den Kontrollvariablen getestet. Zur besseren Nachvollziehbarkeit der Ergebnisse werden die einzelnen Variablen nochmals vorgestellt und in Tabelle 5 zusammengeführt.

Tabelle 5: Variablen der Regressionsmodelle mit Beschreibung

\begin{tabular}{ll}
\hline Variable & Variablenbeschreibung \\
\hline CAAR & CAAR des Zeitfensters -1 bis 1 als Dezimalzahl. \\
log & Natürlicher Logarithmus der Marktkapitalisierung in Mio. US-Dol- \\
(Marktkapitalisierung) & lar 10 Tage vor dem Ereignis. \\
Cashflow & Cashflow des Vorjahres des Unternehmens in Tsd. US-Dollar. \\
Market to Book & 10 Tage vor dem Ereignis. \\
Return on Equity (RoE) & RoE des Vorjahres. \\
Umsatz & Umsatz des Vorjahres in Tsd. US-Dollar. \\
Lead Drug & Dummy $=1$, wenn das Medikament Lead Drug Status hat. \\
Phase & Dummy $=1$, für Phase III und Dummy = 0 für Phase II. \\
Blockbuster & Dummy $=1$, wenn das Medikament Blockbuster-Potential hat. \\
Zulassung & Dummy $=1$, wenn das Medikament bereits eine Marktzulassung \\
Kooperation & hat. \\
Interim & Dummy $=1$, wenn das Medikament in Kooperation erforscht wird. \\
Scheitern & Dummy $=1$, wenn der Rückschlag aus einer Interimsanalyse her- \\
USA & vorgeht. \\
Jahr & Dummy $=1$, wenn das Medikament das erste Mal scheitert. \\
\hline
\end{tabular}

Mit Blick auf die Problematik der Multikollinearität wird in Tabelle 7 im Anhang eine Übersicht der Korrelationen nach Pearson präsentiert. Cohen et al. (2013) haben Konventionen erarbeitet, mit denen sich Korrelationen in unterschiedliche Stufen klassifizieren lassen. Mit 0,92 ist die Korrelation zwischen der Marktkapitalisierung und dem Umsatz als sehr stark einzuschätzen. Daher wird die Variable Umsatz aus dem Regressionsmodell entfernt. Zudem ist die Korrelation zwischen dem Cashflow und der Marktkapitalisierung mit 0,88 sehr hoch, was zum Ausschluss der Variable Cashflow führt. Die restlichen Korrelationen sind als schwach bis moderat einzustufen. Als Kontrollvariablen werden log (Marktkapitalisierung), Cashflow, Market to Book (MtB), RoE, USA, Jahr und Interim genutzt. Aufgrund von nicht verfügbaren Finanzkennwerten werden 23 Ereignisse nicht in die Regressionsanalyse mit einbezogen. Getestet wird das folgende Regressionsmodell, wobei die Variablen in der Klammer jeweils einzeln je Modell hinzugefügt werden:

$C A A R=\alpha+\beta_{1} * \log ($ Marktkapitalisierung $)+\beta_{2} * M t B+\beta_{3} * R o E+\beta_{4} * U S A$

$+\beta_{5} * J a h r+\beta_{6} *$ Interim $+\left(\beta_{7} *\right.$ Scheitern $+\beta_{8} *$ Kooperation $+\beta_{9} *$ Phase

$+\beta_{10} *$ Zulassung $+\beta_{11} *$ Lead Drug $+\beta_{12} *$ Blockbuster $)+\epsilon$ 
Die Ergebnisse der einzelnen Regressionsmodelle sind in Tabelle 6 dargestellt. Das Basismodell, Modell 0, zeigt einen hoch signifikanten positiven Einfluss der Marktkapitalisierung auf die CAAR und unterstreicht nochmals die Befunde der Subsampleauswertungen. Gleiches gilt für die Unterteilung nach Phase II und III. Auch hier werden die Aussagen der Subsamplebetrachtung unterstützt. Die geographische Aufteilung in USA und Rest der Welt besitzt weiterhin keine signifikante Erklärungskraft. Modell 1 stützt die Hypothese, dass es relevant ist, ob das Medikament zum ersten Mal scheitert und bis dato keinen Forschungsrückschlag zu verzeichnen hatte. Die Dummy-Variable Scheitern ist auf dem 0,1\%-Signifikanzniveau hoch signifikant und hat den vermuteten negativen Effekt.

Abgesehen von der Unterstützung der bisherigen Befunde und der Kontrolle der Stationen in der Neuproduktentwicklung zielt die Regressionsanalyse insbesondere auch darauf $\mathrm{ab}$, Forschungsfrage 3 zu untersuchen und zu klären, ob kleinere Unternehmen durch institutionalisierte Designs die Möglichkeit haben, das Verlustpotential im Falle eines Misserfolgs zu begrenzen. Hier ist es naheliegend, das Risiko auf mehrere Schultern zu verteilen und die Medikamentenforschung in Kooperation mit einem Partner durchzuführen. Dementsprechend wird die Dummy-Variable Kooperation in Modell 2 in die Regression einbezogen. Sie ist ebenfalls statistisch signifikant. Das Vorzeichen ist positiv und entspricht damit der postulierten Wirkung aus der Forschungsfrage. Der Kapitalmarkt honoriert somit die Risikoteilung durch Kooperationen mit signifikant kleineren Kursrückschlägen.

Schließlich zeigen die weiteren hinzugefügten Größen die erwarteten Wirkungsrichtungen. Für Lead Drugs, also Medikamente, die in der Entwicklung besonders weit vorangeschritten oder ein Schlüsselprodukt für ein Unternehmen sind, ist ein Scheitern mit schwerwiegenderen Konsequenzen verbunden. Dies gilt tendenziell auch für Medikamente, denen Blockbuster-Potential nachgesagt wird, auch wenn die Befunde hier nicht statistisch signifikant ausfallen.

\section{Diskussion und Fazit}

Neuproduktentwicklungen sind gerade auch für Pharma- und Biotech-Unternehmen, bei denen der Forschungs- und Entwicklungsprozess vergleichsweise transparent abläuft, regelmäßig mit erheblichen Risiken verbunden, die im Falle des Scheiterns auch Konsequenzen für den Aktienkurs und damit die Vermögensposition der Eigentümer haben können. Zur Untersuchung der Kapitalmarktbewertung von veröffentlichten Rückschlägen wurde eine Ereignisstudie präsentiert und ein händisch zusammengestellter, neuer, sehr großer Datensatz mit 224 Rückschlagsankündigungen aus den Jahren 2010 bis 2017 eingesetzt. Im Ergebnis zeigen sich im Zeitraum der Ankündigungen extrem negative Aktienkursreaktionen von durchschnittlich $-23,7 \%$, wobei die Verluste sich nach Größe des Unternehmens markant unterscheiden. Zwar weisen sowohl kleine als auch große Unternehmen signifikant negative Werteffekte auf, der Effekt bei kleinen Unternehmen ist jedoch viel stärker und erreicht durchschnittliche Ausmaße von $-43,7 \%$. Dieses Ausmaß konnte in früheren Studien nicht beobachtet werden.

Potenzielle Erklärungsfaktoren für die Höhe und die Richtung des Werteffekts sind neben der Größe, gemessen als Marktkapitalisierung, u.a. die Phase der klinischen Studien und die Frage der Entwicklung in Kooperation. Demnach lässt sich die Höhe des Werteffektes sowohl durch Unternehmenskennzahlen als auch durch den Inhalt der Veröffentlichung, bzw. den Status der Medikamentenentwicklung, erklären. Für ein kleineres Unternehmen kann vor dem Hintergrund der hier präsentierten Befunde die gezielte Suche nach 
Kooperationspartnern eine Strategie sein, mit der die existentiellen Marktwertverluste im Falle eines Forschungsfehlschlags signifikant reduziert werden können.

Einschränkend und relativierend ist allerdings festzustellen, dass die Wahrscheinlichkeit, dass ein Medikament die klinischen Phasen positiv abschließt, so niedrig ist, dass deutlich mehr Forschungsrückschläge existieren als die mit der Datenerhebung erfassten 263. Andere Studien von Dedman et al. (2008), Ely et al. (2003), Kelm et al. (1995) und Narayanan et al. (2000) bestätigen die verhältnismäßig geringe Anzahl an negativen Meldungen, die publiziert werden. Der weiteren Forschung ist damit angeraten, die Kriterien, die zur Publikation eines Forschungsrückschlags führen, weiter zu analysieren. 


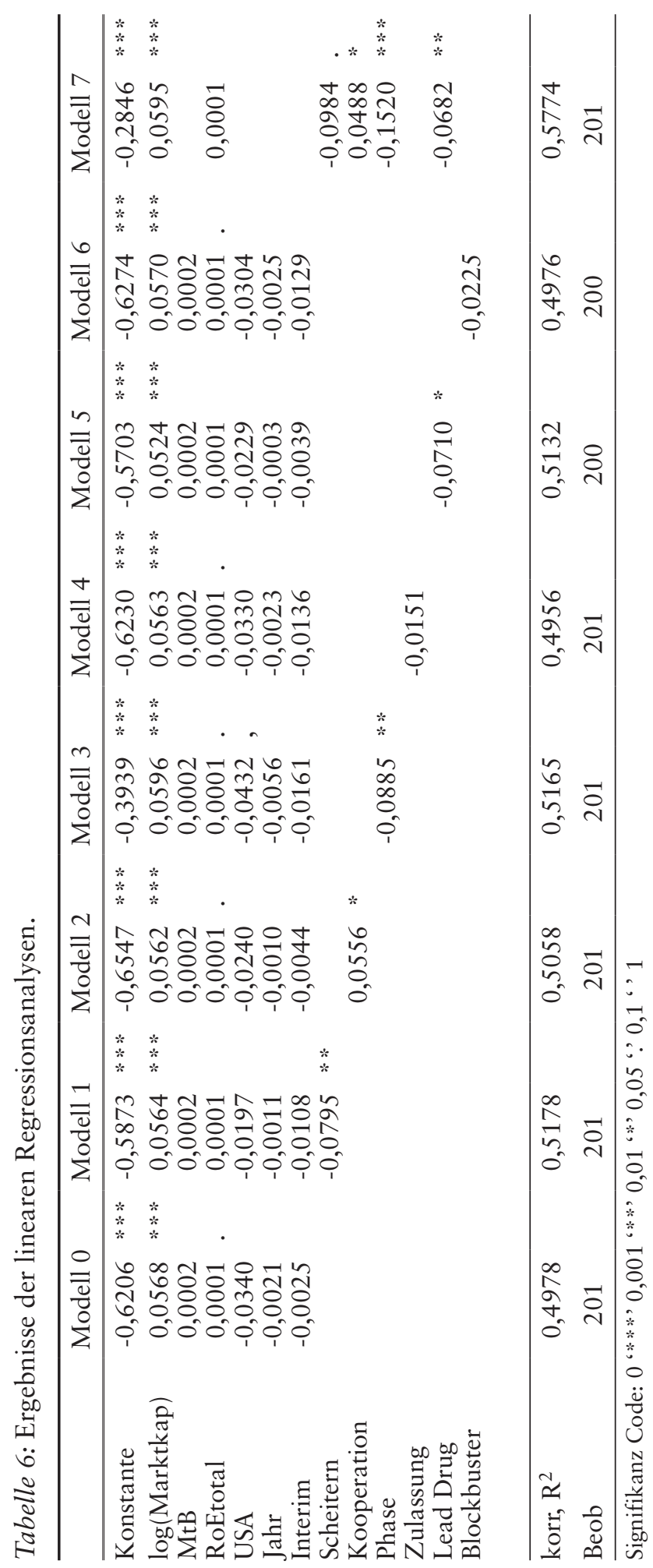




\section{Anhang}

Tabelle 7: Korrelationskoeffizienten.

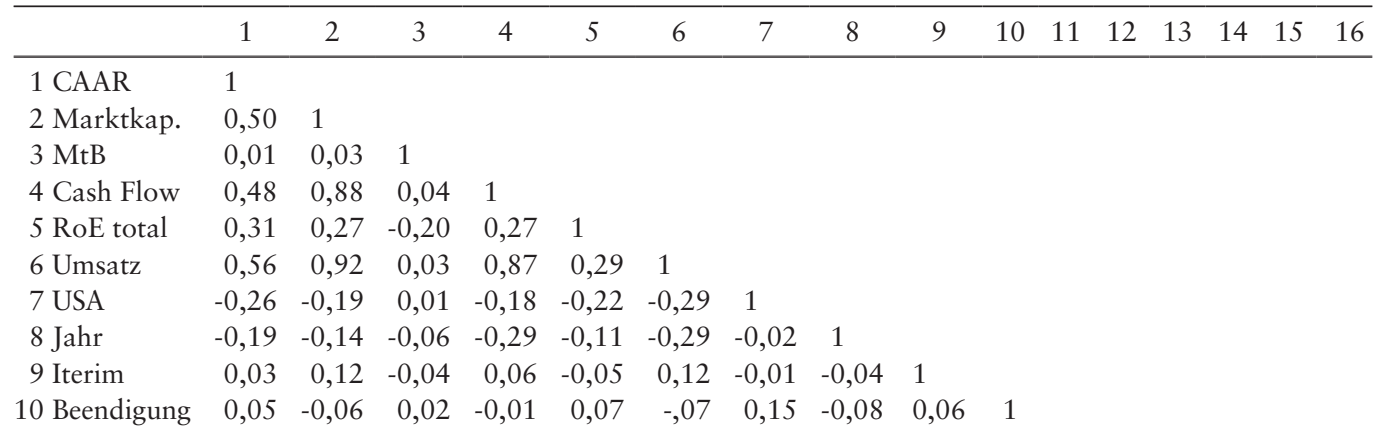

\section{Literaturverzeichnis}

Ahmed, Parvez; Gardella, John; Nanda, Sudhir (2002): Wealth effect of drug withdrawals on firms and their competitors. In: Financial Management 31 (3), S. 21-41.

Black Rock (Hg.) (2018): Fact Sheet iShares Global Helthcare ETF. Online verfügbar unter https://w ww.ishares.com/us/literature/fact-sheet/ixj-ishares-global-healthcare-etf-fund-fact-sheet-en-us.pdf

Boehmer, Ekkehart; Musumeci, Jim; Poulsen, Annette B. (1991): Event-study methodology under conditions of event-induced variance. In: Journal of Financial Economics 30 (2), S. 253-272.

Bosch, Jean-Claude; Lee, Insup (1994): Wealth effects of Food and Drug Administration (FDA) decisions. In: Managerial and Decision Economics 15 (6), S. 589-599.

Chaney, Paul K.; Devinney, Timothy; Winer, Russell S. (1991): The impact of new product introductions on the market value of firms. In: Journal of Business 64 (4), S. 573-610.

Chauvin, Keith W.; Hirschey, Mark (1993): Advertising, R\&D expenditures and the market value of the firm. In: Financial Management 22 (4), S. 128-140.

Chen, Sheng-Syan; Ho, Kim Wai (1997): Market response to product-strategy and capital expenditure announcements in Singapore: investment opportunities and free cash flow. In: Financial Management 26 (3), 82-88.

Chen, Sheng-Syan; Ho, Kim Wai, Ik, Kueh Hwa; Lee, Cheng-few (2002): How does strategic competition affect firm values? A study of new product announcements. In: Financial Management 31 (2), S. 67-84.

Cohen, Louis; Manion, Lawrence; Morrison, Keith (2013): Research methods in education. Routledge.

Dedman, Elisabeth; Lin, Stephen W-J; Prakash, Arun J.; Chang, Chun-Hao (2008): Voluntary disclosure and its impact on share prices -Evidence from the UK biotechnology sector. In: Journal of Accounting and Public Policy 27 (3), S. 195-216.

Deeds, David L.; DeCarolis, Dona; Nelling, Ed (2003): Managing adversity in high technology new ventures: The impact of clinical terminations in the Biotechnology Industry. Online verfügbar unter https://fusionmx.babson.edu/entrep/fer/BABSON2003/II/II-P3/II-P3.html.

DiMasi, Joseph A.; Grabowski, Henry G.; Hansen, Ronald W. (2016): Innovation in the pharmaceutical industry - New estimates of R\&D costs. In: Journal of Health Economics 47 (1), S. 20-33. 
DiMasi, Joseph A.; Hansen, Ronald W.; Grabowski, Henry G. (2003): The price of innovation -New estimates of drug development costs. In: Journal of Health Economics 22 (2), S. 151-185.

Ely, Kirsten; Simko, Paul J.; Thomas, L. G. (2003): The usefulness of biotechnology firms' drug development status in the evaluation of research and development costs. In: Journal of Accounting, Auditing \& Finance 18 (1), S. 163-196.

Fama, Eugene F. (1965): The behavior of stock-market prices. In: Journal of Business 38 (1), S. 34 105.

Grabowski, Henry G.; Kyle, Margaret (2007): Generic competition and market exclusivity periods in pharmaceuticals. In: Managerial and Decision Economics 28 (4-5), S. 491-502.

Grabowski, Henry G.; Vernon, John (2000): The distribution of sales revenues from pharmaceutical innovation. In: Pharmacoeconomics 18 (1), S. 21-32.

Hamill, Philip A.; McIlkenny, Philip; Opong, Kwaku K. (2013): Valuation implications of pharmaceutical companies' R\&D regulatory approval notifications. In: The British Accounting Review 45 (2), S. 99-111.

Hay, Michael; Thomas, David W.; Craighead, John L.; Economides, Celia; Rosenthal, Jesse (2014): Clinical development success rates for investigational drugs. In: Nature Biotechnology 32 (1), S. $40-51$.

$\mathrm{Hu}$, Chengru; Jiang, Wei; Lee, Cheng-few (2013): Managerial flexibility and the wealth effect of new product introductions. In: Review of Quantitative Finance and Accounting 41 (2), S. 273294.

Hwang, Thomas J. (2013): Stock market returns and clinical trial results of investigational compounds -An event study analysis of large biopharmaceutical companies. In: PLOS one 8 (8), S. 18.

Kelm, Kathryn M.; Narayanan, V. K.; Pinches, George E. (1995): Shareholder value creation during R\&D innovation and commercialization stages. In: Academy of Management Journal 38 (3), S. 770-786.

Lin, Wen-Chun; Chang, Shao-Chi (2012): Corporate governance and the stock market reaction to new product announcements. In: Review of Quantitative Finance and Accounting 39 (2), S. $273-$ 291.

MSCI (Hg.) (2017): MSCI World Index (USD). Online verfügbar unter https://www.msci.com/ documents/10199/149ed7bc-316e-4b4c-8ea4-43fcb5bd6523.

Narayanan, V. K.; Pinches, George E.; Kelm, Kathryn M.; Lander, Diane M. (2000): The influence of voluntarily disclosed qualitative information. In: Strategic Management Journal 21 (7), S. 707-722.

Patell, James M. (1976): Corporate forecasts of earnings per share and stock price behavior - empirical test. In: Journal of Accounting Research 14 (2), S. 246-276.

Rothenstein, Jeffrey M.; Tomlinson, George; Tannock, Ian F.; Detsky, Allan S. (2011): Company Stock Prices Before and After Public Announcements Related to Oncology Drugs. In: Journal of the National Cancer Institute 103 (20), S. 1507-1512.

Sarkar, Salil K.; Jong, Pieter J. de (2006): Market response to FDA announcements. In: The Quarterly Review of Economics and Finance 46 (4), S. 586-597.

Schön, Heiko (2015): Pharma M\&A versus alliances and its underlying value drivers: Frankfurt am Main, DOI: 10.3726/978-3-653-05845-1 
Sharma, Anurag; Lacey, Nelson (2004): Linking product development outcomes to market valuation of the firm: The case of the US pharmaceutical industry. In: Journal of Product Innovation Management 21 (5), S. 297-308.

Verband Forschender Arzneimittelhersteller e.V. (Hrsg.) (2015): Forschung für das Leben - Entwicklungsprojekte für innovative Arzneimittel. Online verfügbar unter https:/www.vfa.de/embed/fors chung-fuer-das-leben-2015.pdf.

Niklas Kees, M. Sc., ist Absolvent der Technischen Universität Darmstadt und Student der Hochschule Geisenheim

Anschrift: Straße der Republik 3, 65203 Wiesbaden, NiklasKees@gmx.de

Jochen Panzer, Prof. Dr., ist Professor für Marketing Management an der IUBH.

Anschrift: IUBH Duales Studium, Hanauer Landstraße 147-149, 60314 Frankfurt am Main, j.panzer@iubh.de, +49 (0)69/2547429-21,

Dirk Schiereck, Prof. Dr., ist Universitätsprofessor für Unternehmensfinanzierung an der Technischen Universität Darmstadt.

Anschrift: Technische Universität Darmstadt, Rechts- und Wirtschaftswissenschaften, Fachgebiet Unternehmensfinanzierung, Hochschulstraße 1, 64289 Darmstadt, Schiereck@bwl.tu-darmstadt.de,+49 (0)6151 16-24291 